oxyd-Lösung ohne Benzpyren. Ein Vergleich der Kontrollen der beiden Versuchsgruppen (Abb. 1 und Abb. 2) zeigt, daß die Abnahme der spezifischen Viskosität gering und größenordnungsmäßig die gleiche ist.

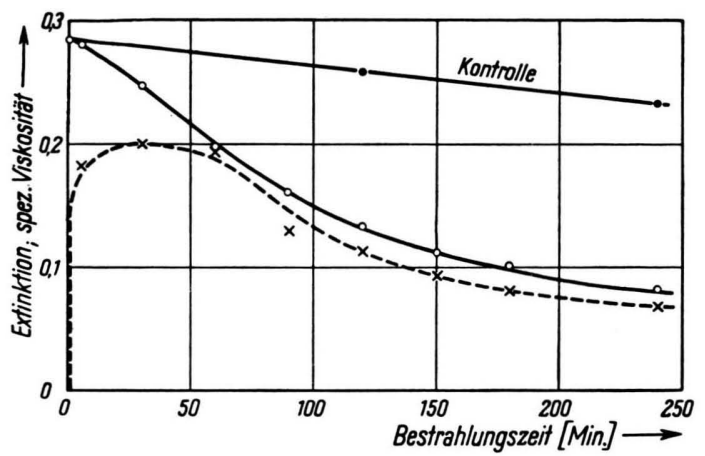

Abb. 2. Der Verlauf von Extinktion und spez. Viskosität in Abhängigkeit von der Bestrahlungszeit nach UV-Einwirkung auf PMAE-Benzpyren-Benzoylperoxyd-Lösungen in Benzol. -...: Extinktion. ०-०: spez. Viskosität. ๑-๑: spez. Viskosität der Kontrollösung (PMAE-Benzoylperoxyd in Benzol).

Das Benzoylperoxyd wirkt, in Ubereinstimmung mit früheren Ergebnissen ${ }^{5}$, lediglich beschleunigend auf den Abbau der Makromoleküle ein.

Die Experimente, bei denen zur PMAE-BenzpyrenLösung zusätzlich Benzoylperoxyd hinzugefügt wurde, lassen vermuten, daß der Sauerstoff bei der hier vorliegenden photosensibilisierten Reaktion eine bedeutende Rolle spielt. In weiteren Versuchen wurde daher die PMAE-Benzpyren-Lösung vor der Bestrahlung in einer geeigneten Quarzküvette (Schichtdicke 7,5 mm) 30 Min. lang mit $\mathrm{O}_{2}$ bzw. gereinigtem $\mathrm{N}_{2}$ durchspült. Eine Abnahme der spezifischen Viskosität konnte lediglich bei der mit $\mathrm{O}_{2}$ durchströmten Lösung festgestellt werden.

Die hier dargestellten Versuche lassen sich durch den von SchönBerg ${ }^{6}$ und SchencK $^{7}$ formulierten Mechanismus der Sauerstoffübertragung mit Hilfe von Sensibilisatoren deuten. Der von uns beobachtete und beschriebene Abbau des Polymers dürfte unter Berücksichtigung der Vorstellungen dieser Autoren durch oxydative Vorgänge ausgelöst werden, bei denen das unter Bestrahlung veränderte Benzpyrenmolekül als $\mathrm{O}_{2}$-Überträger dient. Allerdings mit dem Unterschied, daß sich bei unseren Untersuchungen der Sensibilisator (3.4-Benzpyren) nicht oder nur z. T. wieder zurückbildet. Wie aus den Extinktions-Verläufen und aus dem Verlust der charakteristischen Fluoreszenz hervorgeht, ist mit zunehmender Bestrahlungszeit eine irreversible Veränderung des Benzpyrenmoleküls als wahrscheinlich anzunehmen. Weitere Untersuchungen mit anderen Lösungsmitteln sind im Gange.

5 H. MönIG, Naturwissenschaften 45, 12 [1958].

6 A. Schönberg, Präparative organische Photochemie. Springer-Verlag, Berlin/Göttingen/Heidelberg 1958.

7 G. O. Schenck, s. z. B. Angew. Chem. 69, 579 [1957].

\section{Zur Katalyse der Mutarotation der Glucose durch Ansolvosäuren}

Von W. Broser und G. Ruecker ${ }^{1}$

Institut für Organische Chemie der Freien Universität Berlin, Berlin-Dahlem

(Z. Naturforschg. 15 b, 334-335 [1960]; eingeg. am 30. Dezember 1959)

Die Mutarotation der Glucose wird durch die Ansolvosäuren $\mathrm{AlCl}_{3}$ und $\mathrm{ZnCl}_{2}$ katalysiert; die Katalysekonstanten bei $18^{\circ}$ und $25^{\circ} \mathrm{C}$ und die aus ihnen berechneten Aktivierungsenthalpien und -entropien der katalysierten Reaktion werden mitgeteilt. Die katalytische Wirkung der Ansolvosäuren geht auf die Vergrößerung der Aktivierungsentropie durch den Katalysator zurück.

Bekanntlich ist die Mutarotation der Zucker durch Basen und Protonsäuren katalysierbar ${ }^{2,3}$. Die Katalysekonstanten einer ganzen Reihe von Basen und Protonsäuren wurden ermittelt und in Beziehung zu deren Basen- bzw. Säure-Dissoziationskonstanten gebracht ${ }^{4}$. Dagegen sind im Schrifttum quantitative Angaben über die Katalysierbarkeit der Mutarotation durch Ansolvosäuren nicht vorhanden. Da wir uns im Zusammenhang mit Untersuchungen über den Chemismus der Mutarotation für diese Frage interessierten, führten wir kinetische Versuche durch mit dem Ziel, festzustellen, ob und in welchem Maß die Mutarotation der Glucose durch die Ansolvosäuren $\mathrm{AlCl}_{3}$ und $\mathrm{ZnCl}_{2}$ katalysiert wird.

In Erweiterung der Gleichung von BRoenstedt und Guggenheim ${ }^{4}$ wird der Einfluß der Ansolvosäure $k_{\mathrm{A}}$ auf die direkt meßbare Gesamtkonstante $k$ durch den Ausdruck

$$
k_{\mathrm{A}}=k_{\mathrm{X}} \cdot c_{\mathrm{X}}=k-\left(k_{\mathrm{H}}-k_{\mathrm{p}}\right)
$$

wiedergegeben. $\mathrm{k}_{\mathrm{x}}=k_{\mathrm{x} 1}+k_{\mathrm{x} 2}$ stellt die Summe der katalytischen Konstanten der Hin- und Rückreaktion der Ansolvosäure, $c_{\mathrm{x}}$ deren Konzentration dar. Die Größen $k_{\mathrm{H}}$ und $k_{\mathrm{p}}$, die den Einfluß des Wassers und der Hydroniumionen bzw. des Puffers berücksichtigen, sind rechnerisch zugänglich. Aus $k_{\mathrm{x}}$ und der Gleichgewichtskonstante $K$ lassen sich $k_{\mathrm{x}_{1}}$ und $k_{\mathrm{x} 2}$ und aus deren Temperaturabhängigkeit in bekannter Weise die Aktivierungsenthalpie $\Delta H_{\mathrm{z}}{ }^{\circ}$ und die Aktivierungsentropie $\Delta S_{\mathrm{z}}{ }^{\circ}$ berechnen (Einzelheiten s. 1. c. ${ }^{1}$ ).

Die Geschwindigkeit der Mutarotation wurde bei $18,0^{\circ} \mathrm{C}$ und $25,0^{\circ} \mathrm{C}$ polarimetrisch gemessen. Die Konzentration an D-Glucose betrug stets $0,222 \mathrm{Mol} / l$. Um die Gleichgewichtseinstellung von beiden Seiten aus zu verfolgen, wurde bei den Messungen sowohl von $\alpha$-, als auch $\beta$-D-Glucose ausgegangen. Die Lösungen enthielten zur Pufferung $0,552 \mathrm{Mol} / l$ Essigsäure und $0,08 \mathrm{~mol} / l$ Natriumhydroxyd. Der $p_{\mathrm{H}}$-Wert der ansolvosäure-freien Lösungen betrug bei $18{ }^{\circ} \mathrm{C} 3,70$, bei $25^{\circ} \mathrm{C} 3,74$. Es

1 G. RückER, Diplom-Arbeit, F.U. Berlin 1958.

2 Hesse, Liebigs Ann. Chem. 76, 101 [1875].

3 T. Osaka, Z. physik. Chem. 35, 661 [1900].

4 I. N. Brönstedt u. E. A. Guggenheim, J. Amer. chem. Soc. 49, 2554 [1927]. 


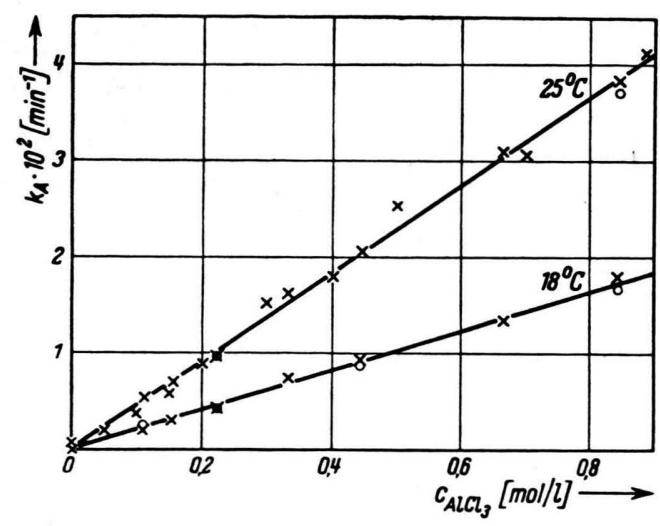

Abb. 1. Katalyse durch $\mathrm{AlCl}_{3}$. ( $0=\beta$-Glucose, $x=a$-Glucose) .

wurden Versuchsreihen mit steigendem Ansolvosäuregehalt durchgeführt. Der $p_{\mathrm{H}}$-Wert der Lösung wurde nach Einstellen des Gleichgewichtes potentiometrisch gemessen und zur Berechnung von $k_{\mathrm{H}}$ und $k_{\mathrm{p}}$ herangezogen. Er verschiebt sich mit steigender Ansolvosäure-Konzentration zu kleineren Werten.

Die Abb. 1 und 2 geben die Konzentrations-Abhängigkeit von $k_{\mathrm{A}}$ wieder. Die Meßwerte liegen auf Geraden, aus deren Steigungen durch Ausgleichsrechnung nach der Methode der kleinsten Fehlerquadrate $k_{\mathrm{x}}$ und dessen mittlerer Fehler bestimmt wird. Da besondere Versuche ergeben haben, daß im untersuchten Bereich die Gleichgewichtskonstante temperatur-un-abhängig ist, kann in allen Fällen der Wert $K=1,76^{5}$ zur Berechnung von $k_{\mathrm{x}_{1}}$ und $k_{\mathrm{x} 2}$ herangezogen werden. Zum Vergleich wurde unter denselben Bedingungen die Beeinflussung der Mutarotation durch $\mathrm{MgCl}_{2}$ und $\mathrm{NaCl}$ gemessen. Wie man aus Tab. 1 erkennt, in der die Ergebnisse zusammengestellt sind, katalysiert Kochsalz überhaupt nicht, während die katalytische Konstante des $\mathrm{MgCl}_{2}$ nur ein Bruchteil der der Ansolvosäuren be-

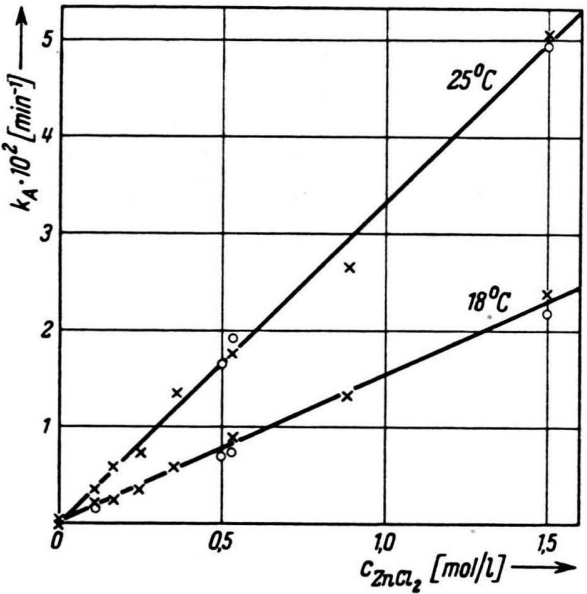

Abb. 2. Katalyse durch $\mathrm{ZnCl}_{2}$. ( $\mathrm{O}=\beta$-Glucose, $\mathrm{x}=\alpha$-Glucose $)$.

trägt. Dies berechtigt zu dem Schluß, daß die Katalyse von $\mathrm{AlCl}_{3}$ und $\mathrm{ZnCl}_{2}$ nicht auf einem "Salzeffekt", sondern auf ihrer Eigenschaft als Ansolvosäure beruht. In Tab. 1 sind außerdem noch die aus Literaturangaben berechneten katalytischen Konstanten für Wasser, Hydroniumion sowie undissoziierter Essigsäure und Acetation angeführt. Bemerkenswert ist die Tatsache, daß die Aktivierungsenthalpien der Ansolvosäuren größer sind als die des Wassers und zwar desto größer, je besser sie katalysieren; d. h. die Beschleunigung der Mutarotation beruht ausschließlich auf der Vergrößerung der Aktivierungsentropie.

Weiterreichende Schlüsse im Hinblick auf den Chemismus der Mutarotation können erst nach Vorliegen weiterer Versuchsergebnisse, mit deren Erarbeitung wir z. Zt. beschäftigt sind, gezogen werden.

Herrn Prof. Dr. W. Lautsch sei an dieser Stelle für die Förderung dieser Arbeit, dem "Fnnds der Chemischen Industrie “ für die Bereitstellung von Mitteln gedankt.

\begin{tabular}{|c|c|c|c|c|c|c|}
\hline Katalysator & $\begin{array}{c}k_{\mathrm{x}}^{18} \cdot 10^{2} \\
{[1 / \mathrm{min} \cdot \mathrm{Mol}]}\end{array}$ & $\begin{array}{c}k_{\mathrm{x}_{1}}^{18} \cdot 10^{2} \\
{[1 / \mathrm{min} \cdot \mathrm{Mol}]}\end{array}$ & $\begin{array}{c}k_{\mathrm{x}}^{25} \cdot 10^{2} \\
{[1 / \mathrm{min} \cdot \mathrm{Mol}]}\end{array}$ & $\left|\begin{array}{c|}k_{\mathrm{x}_{1}}^{25} \cdot 10^{2} \\
{[1 / \mathrm{min} \cdot \mathrm{Mol}]}\end{array}\right|$ & $\begin{array}{l}\Delta H_{\mathrm{z}_{1}}^{\circ} \\
{[\mathrm{kcal}]}\end{array}$ & $\begin{array}{l}\Delta S_{\mathrm{z}_{1}}^{\circ} \\
{[\mathrm{Cl}]}\end{array}$ \\
\hline $\begin{array}{l}\mathrm{AlCl}_{3} \\
\mathrm{ZnCl}_{2} \\
\mathrm{MgCl}_{2} \\
\mathrm{NaCl}^{2}\end{array}$ & $\begin{array}{r}2,05 \text { ஜ } \\
1,53 \text { @ } \\
0,45 \stackrel{H}{-0,03}\end{array}$ & $\begin{array}{r}1,31 \text { 응 } \\
0,98 \text { ᄋ } \\
0,29 \text { H } \\
-0,02\end{array}$ & 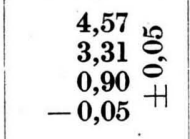 & $\begin{array}{r}2,91 \\
2,11 \\
0,57 \\
-0,03\end{array}$ & $\begin{array}{c}19,1 \pm 0,5 \\
18,4 \pm 0,6 \\
16,5 \pm 1,7 \\
-\end{array}$ & $\begin{array}{c}-9,4 \pm 1,5 \\
-12,6 \pm 1,9 \\
-21,6 \pm 6 \\
\pm\end{array}$ \\
\hline $\begin{array}{l}\mathrm{H}_{2} \mathrm{O} \\
\mathrm{H}_{3} \mathrm{O} \oplus \\
\mathrm{CH}_{3} \cdot \mathrm{COOH} \\
\mathrm{CH}_{3} \cdot \mathrm{COO} \odot\end{array}$ & $\begin{array}{c}0,022^{4} \\
33,39^{4} \\
0,46^{4} \\
6,22^{4}\end{array}$ & $\begin{array}{c}0,014 \\
21,29 \\
0,29 \\
3,97\end{array}$ & $\begin{array}{c}\mathbf{0 , 0 4 0 ^ { 6 }} \\
\mathbf{5 9 , 4 2 ^ { 6 }} \\
- \\
-\end{array}$ & $\begin{array}{c}0,026 \\
37,89 \\
- \\
-\end{array}$ & $\begin{array}{r}14,1 \\
13,6 \\
- \\
-\end{array}$ & $\begin{array}{r}-35,7 \\
-22,9 \\
- \\
-\end{array}$ \\
\hline
\end{tabular}

Tab. 1. Zusammenstellung der Ergebnisse.

5 H. S. Isbell u. W. W. Pigman, J. Res. nat. Bur. Standards 18, 141 [1937].
6 C. S. Hudson, J. Amer. chem. Soc. 29, 1571 [1907]. 